\title{
A comparison of different fractal analyses for VHF electromagnetic emissions and their self-organization for the off-sea Miyagi-prefecture earthquake
}

\author{
N. Yonaiguchi ${ }^{1}$, Y. Ida ${ }^{1}$, M. Hayakawa ${ }^{1}$, and S. Masuda ${ }^{2}$ \\ ${ }^{1}$ The University of Electro-Communications, Department of Electronic Engineering and Research Station on Seismo \\ Electromagnetics, 1-5-1 Chofugaoka, Chofu Tokyo 182-8585 Japan \\ ${ }^{2}$ Tohoku Intelligent Communications Co., Ltd., Sendai, Japan
}

Received: 24 May 2007 - Revised: 8 August 2007 - Accepted: 20 August 2007 - Published: 24 August 2007

\begin{abstract}
The VHF electromagnetic noise intensity data at several stations in the Tohoku area of Japan during the period of a rather large (with magnitude of 7.2) earthquake (Miyagi-ken oki earthquake) taken place on 16 August 2005, are analyzed by means of different fractal analysis methods, including (1) spectral slope estimation, (2) multi-fractal detrended fluctuation analysis and (3) multi-fractal wavelet transform modulus maxima method. It seems to the authors that there is no definite analysis method for the analysis of any seismogenic phenomenon, so that the only way we have to take, is to apply different methods to the same data for the detailed comparison of the results. This comparison enables us to deduce the properties commonly observed by the above methods. Because the most important feature common to these three methods, is that significant changes in fractal scaling characteristics are observed just during the earthquake (mainly before the earthquake) only at one station of Kunimi. Finally, we can come to the definite conclusion on the self-organization of VHF emissions only at one station in the present case.
\end{abstract}

\section{Introduction}

An earthquake (EQ) is a large-scale fracture phenomenon in the Earth's heterogeneous crust and a vital problem in the short-term EQ prediction is the identification of precursors of EQs. When a heterogeneous material like Earth's crust is strained, its nonlinear evolution toward the final rupture is characterized by self-organization toward the crtical stage including the local nucleation and coalescence of microcracks (i.e., self-organized criticality (SOC)). Both acoustic and electromagnetic emissions in a wide frequency range

Correspondence to: M. Hayakawa

(hayakawa@whistler.ee.uec.ac.jp) from DC, ULF up to VHF, are produced by microcracks, which can be considered as the so-called precursors of the general rupture of EQs (see the monographs by Hayakawa and Fujinawa (1974), Hayakawa (1999), and Hayakawa and Molchanov (2002)).

Such a nonlinear dynamics taking place in Earth's crust toward the rupture (EQ), can be satisfactorily investigated with the use of fractal analysis. Hayakawa et al. (1999) performed the first attempt of fractal analysis for ULF emissions based on their spectral slope analysis, and found that a significant change was observed a few weeks to a few days before the EQ. Later, different kinds of analysis methods have been developed by Smirmova et al. (2001, 2004), Gotoh et al. (2002, 2004), and Ida and Hayakawa (2006). Recently, Ida et al. $(2005,2006)$ have extended the previous monofractal analysis to multi-fractal one, in order to know the fine structure changes tacking place just before the EQ. Then, this kind of fractal analysis has been rather popular in other frequency ranges as well, including DC emissions (SES (seismic electric signals) (Varotsos, 2005)), and VHF/VLF emissions (e.g. Eftaxias et al., 2002; Kapiris et al., 2002, 2004).

Electromagnetic noises in the frequency range of VHF $(49.5 \mathrm{MHz})$ are known to consist of a few noise sources (Degauque and Hamelin, 1993); (1) atmospheric noise (due to lightning), (2) man-made composite noise, (3) solar-terrestrial noise (form the Earth's magnetosphere/ionosphere, solar system, cosmic noise etc.), and (4) any other noise such as lithospheric effect. The last lithospheric effect of VHF emissions, is known to follow the SOC process as a precursory effect of an EQ, so that the fractal analysis in this paper is used to sort out only this lithospheric effect. This kind of VHF lithospheric emissions has been recently reported by Kapiris et al. (2002), but the generation mechanism of those VHF emissions is extremely poorly understood. In the case of lithospheric ULF emissions, they are believed to be generated near the hypocenter of an EQ

Published by Copernicus Publications on behalf of the European Geosciences Union. 


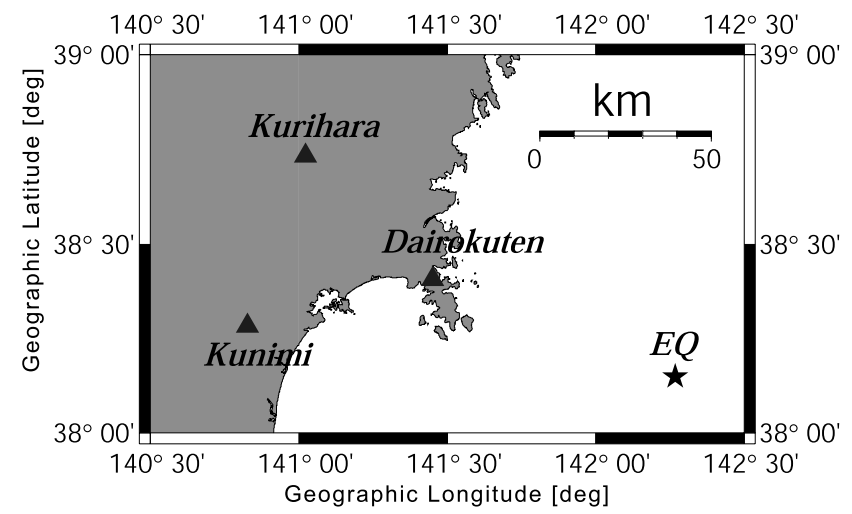

Fig. 1. Relative location of the epicenter (as indicated by a cross in the sea) and three representative VHF observing stations (Dairokuten, Kurihara and Kunimi). The distance between each two of 3 stations is approximately $50 \mathrm{~km}$, and the epicentral distance from Dairokuten is about $90 \mathrm{~km}$.

either by the mechanism of microfracturing (Molchanov and Hayakawa, 1995) or electro-kinetic effect (Fenoglio et al., 1995). Unlike this ULF emission, due to the severe attenuation in the lithosphere VHF emission cannot be detected on the Earth's surface even if they are generated in the EQ hypocenter. However, we have to think of the secondary effect in such a way that those VHF emissions are generated in the Earth's surface or in the atmosphere due to the precursory tectonic effect of an EQ.

This paper is intended to supplement our recent paper by Yonaiguchi et al. (2007), in which they have studied the nonlinear process for seismogenic VHF noise using only the multi-fractal detrended analysis. In order to provide further support to the result by Yonaiguchi et al. (2007), we try to show the results by using different kinds of fractal analysis and comparing those results in order to validate their previous results.

\section{VHF data used in this paper}

The EQ for our analysis took place at $11 \mathrm{~h} 46 \mathrm{~m} 25 \mathrm{~s} \mathrm{L.T.} \mathrm{on}$ 16 August 2005, with the name of Miyagi-ken oki EQ (Offsea of Miyagi prefecture). Its magnitude was 7.2 (a rather large EQ in this area) with the depth of $42 \mathrm{~km}$. Its epicenter is located at the geographic coordinates $\left(38.1^{\circ} \mathrm{N}, 142.4^{\circ} \mathrm{E}\right)$, as indicated as a cross in Fig. 1.

We have seven observing stations for VHF natural electromagnetic noises in the Tohoku area around Sendai, but only three representative VHF stations (Dairokuten, Kurihara and Kunimi) are illustrated in Fig. 1 and used for analysis. The distance between any pair of two stations is about $50 \mathrm{~km}$, and the distance between Dairokuten and the epicenter is approximately $90 \mathrm{~km}$. We will present the time series data of VHF electromagnetic noises during a sufficiently long period (about one month before and one month after the EQ). Next, we describe the VHF observing system. The observing antenna is a discon-type (wideband characteristics from 25 to $1300 \mathrm{MHz}$ ) installed on the top of a tower at each station, but the observing frequency is tuned to $49.5 \mathrm{MHz}$ in the radio receiver. The intensity recorded at each station, is transmitted to the master station in Sendai.

The most important point of this paper is (1) multistationed network and (2) longer period of analysis as compared with the paper by Eftaxias et al. (2002). First of all, we have to point out one important aspect of the data comparing the VHF data at seven stations. It is found that the VHF data at the three stations of Dairokuten, Kurihara and Kunimi are completely different from each other, which is importantly indicative of a highly localized nature of VHF emissions. Also, there is no clear diurnal variation in the VHF intensity variation, being very different from lower frequency emissions (such as ULF, ELF and VLF).

\section{Fractal analysis of VHF emissions}

As is shown in Yonaiguchi et al. (2007), the use of fractal analysis enables us to sort out only the lithospheric VHF emissions organized by the SOC process among a few possible noise sources because other noise sources do not follow the SOC process.

There have been proposed several methods of fractal analysis to study the nonlinear dynamics, but we have used the following few methods which have been conventionally used; (1) Estimation of slope in frequency spectrum, (2) MFDFA (multi-fractal detrended fluctuation analysis), (Kantelhardt et al., 2002), and (3) WTMM (Wavelet transform modulus maxima) (Muzy et al., 1993; Mallat and Hwang, 1992). The latter two methods are competitive to detect the multifractality. MFDFA is based on the identification of scaling of the qth-order moments whose power-law depends on the signal length. The other WTMM consists in detection of scaling of the maximum lines of the continuous wavelet transform on different scales in the time-scale plane. First, we present the result of analysis by each method, and then we compare those results in order to obtain reliable information on the lithospheric process.

\subsection{Spectral slope method and results}

An important feature that characterizes the dynamics in a heterogeneous medium close to its final failure lies in the appearance of memory effects. If the time series of amplitude of the VHF electromagnetic emission $A\left(t_{i}\right)$ is a temporal fractal, then a power-law spectrum density of the recorded time series is expected,

$S(f)=\alpha f^{-\beta}$.

The spectral amplification factor $\alpha$ in Eq. (1) indicates the power of the spectral components. The linear correlation coefficient $r$ is a measure of the quality of fit of the observed 

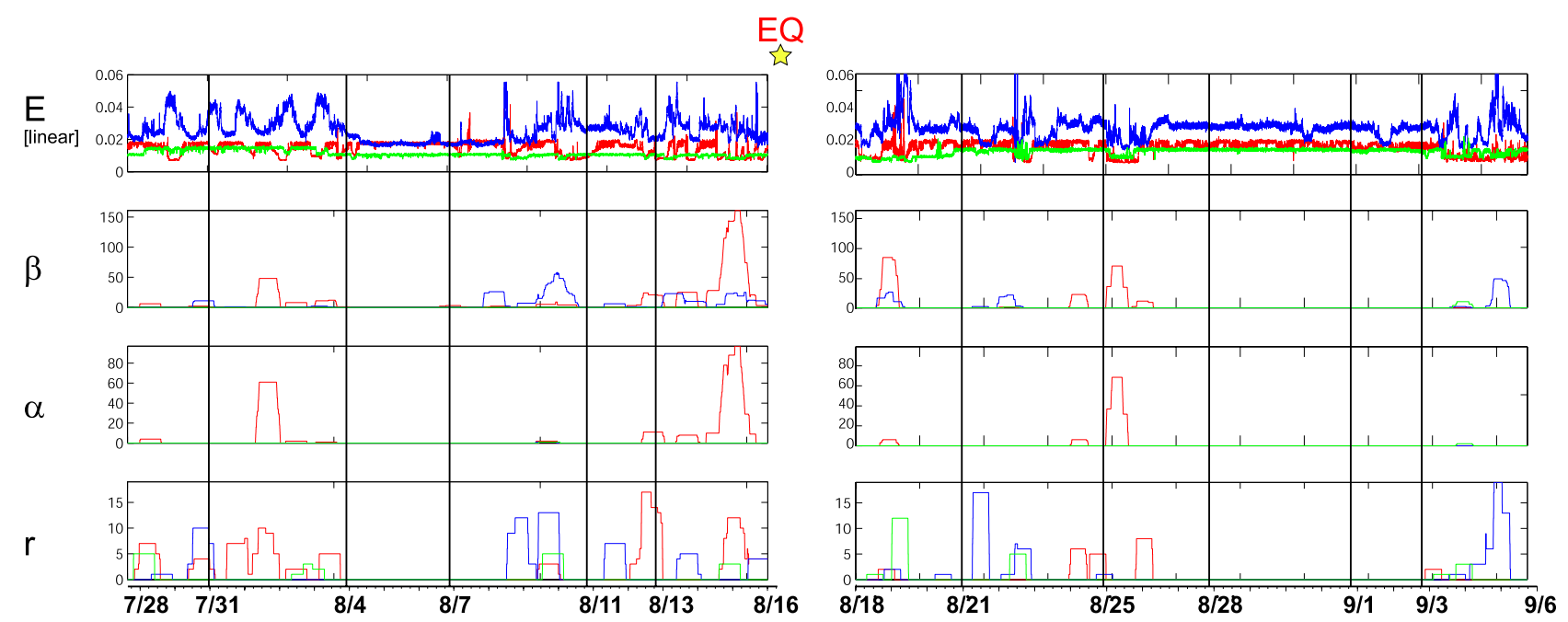

Fig. 2. The result of VHF electromagnetic noises by means of spectral slope analysis. The top indicates the temporal variation of VHF signal at these stations (Dairokuten in Blue, Kurihara in green and Kunimi in Red). The lower three panels refer to the temporal variations of $\beta$, $\alpha$ and $\gamma$ (in the form of occurrence number with $\beta \geq 2.5, \alpha \geq 9.0$ and $\gamma \geqq 0.97$ ). The color indicates the station.

power spectral density to the theoretical power law given by Eq. (1). The wavelet transform with the use of Morlet mother function, was used for the decomposition of the signals.

Figure 2 illustrates the results by this method. The left part refers to the period before the $\mathrm{EQ}$, while the right part after the EQ. The color is defined to correspond to each of the three stations in Fig. 1; Blue, Dairokuten, green, Kurihara and red, Kunimi. We here follow the way adopted by Kapiris et al. (2004), and Fig. 2 is the result on the number of events based on the following criteria; $\beta>2.5, \alpha>9.0$ and $r>0.97$.

Figure 2 indicates that the quantities in green (at Kurihara) do not exhibit any significant variations. So that, we will compare only the two curves in blue and red. First, we look at the variation of $r$ in order to know how good the observed spectral density fits with the power-law. The variation of $r$ for the station of Kunimi (in red) in the bottom panel of Fig. 2 in the form of occurrence number, indicates a significant change before and after the EQ. That is, the occurrence rate of $r>0.97$ takes place from 28 July to 4 August and 12 August to just before the EQ (16 August). And after the EQ there is no significant period of highly enhanced occurrence of $r \geq 0.97$ till 24 August. Generally speaking, it is clear that the number of time intervals with $r>0.97$ increases progressively as the main shock (EQ) is approached, and the powerlaw behavior breaks down after the EQ. That is, a time region with $r$ close to unity is approached during the last few days of the EQ.

Correspondingly to the enhancement in $\mathrm{r}$ before the EQ, the occurrences of $\alpha>9.0$ and $\beta>2.5$ are seen to increase a few days before the EQ. While, the occurrence in $\beta$ seems to decrease after the EQ. On the other hand, when we look at the variations at other stations (Dairokuten in blue and Kurihara in green). We can notice some changes, for example, an enhancement in the occurrence number of time intervals with $r \geq 0.97$ a few weeks to just before the EQ and also one week after the EQ. However, we cannot identify any significant changes in the upper two panels in Fig. 2 ( $\alpha$ and $\beta$ values), which is indicating that self-organization is NOT taking place in the VHF data at Dairokuten (in blue). Only, significant changes are clearly identified for the station of Kunimi (in red), where the VHF signals are self-organized before the EQ. The temporal evolution at Kunimi seems to be in good agreement with Eftaxias et al. (2002).

\subsection{Multi-fractal detrended fluctuation analysis (MF-DFA) and results}

The VHF noise data as a time series are sampled every $100 \mathrm{~s}$ (before the EQ) or $72 \mathrm{~s}$ (after the EQ), and we apply the multi-fractal analysis for those VHF data. Multi-fractal analysis is known to provide us with much more information than the simple mono-fractal analysis (Ida et al., 2005, 2006).

In this section we first present the result by the MF-DFA (not standard, but extended). The MFDFA is apparent to be much more accurate than the conventional box counting, and the detrended fluctuation analysis (DFA) has become a widely-used technique for the determination of fractal scaling properties and the detection of long-range correlations in noisy, nonstationary time series (Gotoh et al., 2004). One reason to employ the DFA method is to avoid spurious detection of correlations that are artifacts of nonstationarities in the time series. We followed the analysis of DFA method developed by Kantelhardt et al. (2002). Let us suppose that $x_{k}$ is a time series of length $N$, and we first determine the 


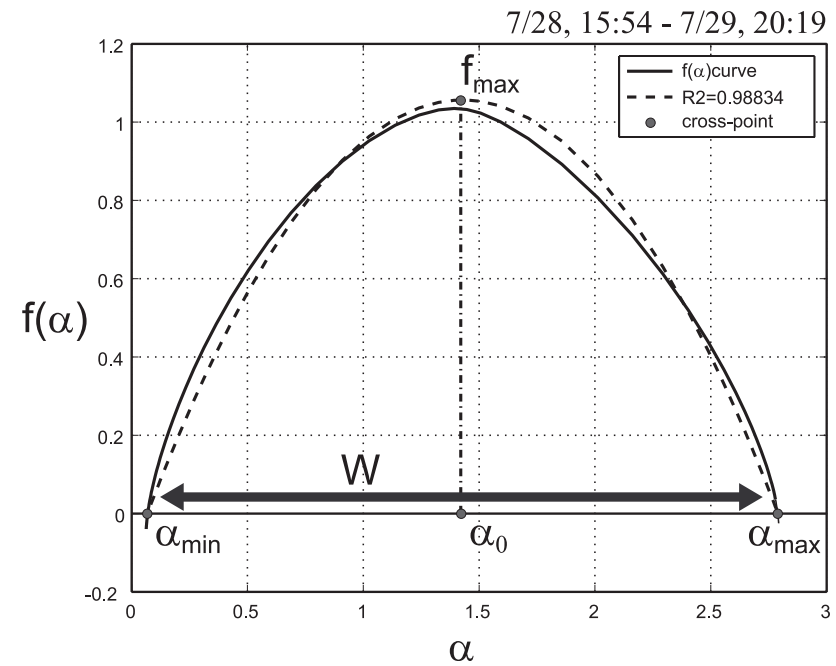

Fig. 3. An example of obtained $f(\alpha)$ curve of the VHF emissions during a period of 15:54 LT on 28 July to 20:19 LT on 29 July at the station of Kunimi. The full line is observed $f(\alpha)$ and the broken line is its corresponding approximated $f(\alpha)$. The definitions of $\alpha_{\max }, \alpha_{\min }, w\left(\equiv \alpha_{\max }-\alpha_{\min }\right), \alpha_{0}$ and $f_{\max }$ are given.

profile

$Y(i) \equiv \sum_{k=1}^{i}\left[x_{k}-\langle x\rangle\right] \quad(i=1, \cdots, N)$

(where $\langle x\rangle$ is the mean). Next we divide the profile $Y(i)$ into $N_{s} \equiv \operatorname{int}(N / s)$ (int means integer) non-overlapping segments of equal length s. Since the length $N$ of the series is often not a multiple of the considered time scales, a short part at the end of the profile may remain. In order not to disregard this part of the series, the same procedure is repeated starting from the opposite end. Thereby, $2 N_{s}$ segments are obtained altogether. Then, we calculate the local trend for each of the $2 N_{s}$ segments by a least-square fit of the series and we determine the variance

$F^{2}(v, s) \equiv \frac{1}{s} \sum_{i=1}^{s}\left\{Y[(v-1) s+i]-y_{v}(i)\right\}^{2}$

for each segment $v\left(v=1, \ldots, N_{s}\right)$ and

$F^{2}(v, s) \equiv \frac{1}{s} \sum\left\{Y[(v-1) s+i]-y_{v}(i)\right\}^{2}$

for $v=N_{s}+1, \ldots, 2 N_{s}$. Here, $y_{v}(i)$ is the fitting polynomial in segment $v$. We have used the sixth-order $(m=6)$ polynomials in the fitting procedure. We then average over all segments to obtain the $q$-th order fluctuation function.

$F q(s) \equiv\left\{\frac{1}{2 N_{s}} \sum\left[F^{2}(v, s)\right]^{\frac{q}{2}}\right\}^{\frac{1}{q}}$

Of course, $F q(s)$ depends in the DFA order $\mathrm{m}$, and by construction $F q(s)$ is only defined for $s \geq m+2$. Kantelhardt et al. (2002) have suggested the value for $s$ in such a way that $s<N / 4$. So that we have adopted a reasonable range of $s=10 \sim 300$, and so the obtained results are acceptable. We obtain the $s$ dependence of $F q$, which indicates that $F q(s)$ decrease as a power law for large values of $s$ if the series $x_{k}$ are long-range power-law corrected, and we can estimate the $h(q)$, the generalized Hurst exponent. It is seen that $F q(s)$ can be approximated as $\propto s^{\tau(q)}$, so that we can estimate the $\tau(q)$.

On the basis of obtained $\tau(q)$ characteristics, we can estimate the well-known curves of $f(\alpha)-\alpha$ (where $\alpha$ is the Lipschitz-Hölder exponent) and $D q$ curve by means of the following relationships

$\alpha(q)=d \tau(q) / d q$

$f(\alpha(q))=q \alpha(q)-\tau(q)$

$D(q)=D q=\tau(q) /(q-1)$

(see the textbooks by Goltz (1997) and Feder (1988)).

We show one example of $f(\alpha)$ curve for a particular time interval from 15:54 on 28 July to 20:19 on 29 July before the EQ in Fig. 3. The sampling before the EQ is $100 \mathrm{~s}$ (as one point), so that we took 1024 points (this corresponds to the above time interval of about $28 \mathrm{~h}$ ). By using these data, the full line in Fig. 3 is obtained by the extended MF-DFA method. The general form of this $f(\alpha)$ is very regular, so that we can estimate very easily the multi-fractal parameters. However, when we deduce the $f(\alpha)$ curve, we have sometimes some irregularity in the $f(\alpha)$ curve and we wonder whether we have to accept such a $f(\alpha)$ curve or not. So we have made the following criterion: When the obtained $f(\alpha)$ curve as in a full line in Fig. 3 is approximated by a quadratic equation of $\alpha$ with correlation function exceeding 0.95, we accept the $f(\alpha)$ curve and we use the approximated $f(\alpha)$ curve to deduce the multi-fractal parameters. First, $\alpha_{\max }$ and $\alpha_{\min }$ are obtained from the relation of $f(\alpha)=0$, and the width $w$ is defined as $w\left(\equiv \alpha_{\max }-\alpha_{\min }\right) . \quad F(\alpha)$ takes a maximum value $f_{\max }$ at a specific $\alpha_{0}$. In Fig. 3, the correlation is found to be 0.988 , well above 0.95 .

Figure 4 is the overall summary of the multi-fractal analysis of VHF emissions at the three stations. The occurrence time of the EQ is indicated by a EQ star; the left part of Fig. 4 refers to the period before the EQ, while the right part, the period after the EQ. The color corresponds to a station: blue line refers to Dairokuten, green, Kurihara and red, Kunimi as shown in Fig. 2. The top panel in Fig. 4 illustrates the electric field intensity of observed VHF emissions. Below we illustrate the parameters of multi-fractality; from the top, $w\left(\equiv \alpha_{\max }-\alpha_{\min }\right), \alpha_{\max }, \alpha_{\min }, \alpha_{0}$ and finally the nonuniformity factor $\Delta\left(=w / f_{\max }\right)($ Goltz, 1997). The color is the same as in the top panel. First of all we look at the temporal evolutions of VHF emission intensity. The VHF activity at all of the three stations are seen to be very stable after the EQ. While, we observe relatively enhanced VHF activity 

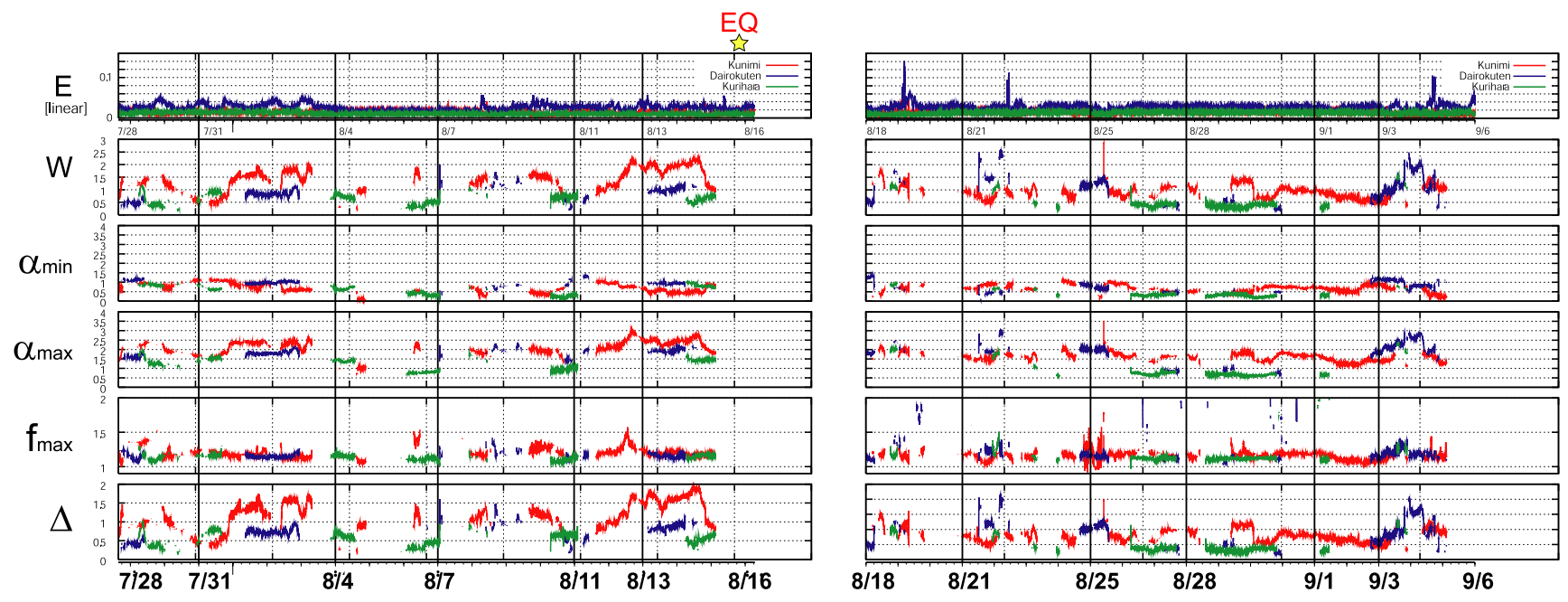

Fig. 4. Temporal evolutions of VHF radio emissions and their corresponding multi-fractal quantities by means of MF-DFA. EQ means the time of the EQ or 16 August, 2005. The left part refers to the period before the EQ, while the right, after the EQ. The top panel indicates the real VHF emissions intensity at three stations (Blue Dairokuten, green Kurihara and red Kunimi). Below the parameters of multi-fractality are given from the top, $w\left(\equiv \alpha_{\max }-\alpha_{\min }\right), \alpha_{\max }, \alpha_{\min }, \alpha_{0}$ and $\Delta\left(\equiv w / f_{\max }\right)$ (non-uniformity factor). The same color is valid for each station.

before the EQ. But, 3 stations exhibited considerably different features. The VHF noise at Kurihara (in green) is still at a low level, without any significant enhancement. On the contrary, the VHF intensity at Dairokuten (in blue) is considerably enhanced with some modulations during the period of 28 July to 3 August. And we have a quiet period from $4 \mathrm{Au}-$ gust to 8 August, Then, again we have a prolonged period of high activity from 8 August to just before the EQ. The intensity at Kunimi in red, showed the similar general tendency. When we look at the parameters of multi-fractality in the lower panels in Fig. 4, we notice that the points are not continuous with time. We have to explain why we have such discontinuities in the multi-fractal parameters. When we presented one example in Fig. 3, we reject the $f(\alpha)$ curve obtained when the $f(\alpha)$ curve is not good enough to have the correlation coefficient less than 0.95 . The computation of multi-fractal parameters is performed for one data with 1024 points, with shifting one point ( $100 \mathrm{~s}$ before the EQ and $72 \mathrm{~s}$ after the EQ). Where we have no point in the multi-fractal analysis in Fig. 4, we have not good $f(\alpha)$ curves.

We examine, in Fig. 4, the temporal behavior of the multifractal parameters at each station, one by one. The first three parameters $\left(w\left(\equiv \alpha_{\max }-\alpha_{\min }\right), \alpha_{\max }\right.$ and $\left.\alpha_{\min }\right)$ are interrelated, so that we deal with these together. The most important and seemingly significant point at Dairokuten (in blue) is that even though the VHF noise intensity is considerably enhanced for some periods, both of $\alpha_{\min }$ and $\alpha_{\max }$ (correspondingly $w$ ) are seen to be stable during the whole period before and after the EQ. Also, the value of $\alpha_{o}$ with $f_{\max }$ (5th panel) remains just around $\alpha_{o} \approx 1.25$ during the whole period. The most significant parameter $\left(\Delta\left(=w / f_{\max }\right)\right)$; nonuniformity factor remains stable in a range of $\Delta \cong 0.5-1.0$.
Then, we consider the data at Kurihara (given in green). The VHF noise there is extremely low, without any significant enhancement (though the close inspection indicates the enhancement before the EQ as compared with that after the EQ). The multi-fractal parameters $\left(w, \alpha_{\max }, \alpha_{\min }\right.$ and $\left.\alpha_{o}\right)$ are seen to exhibit no significant changes, together with the $\Delta$ (at the bottom). If we suppose that the VHF emissions at Dairokuten (in blue) were due to the EQ, we would expect significant precursory (self-organization) effects before the EQ, which is in complete discrepancy with the results at Dairokuten. This suggests that the enhanced VHF noises observed at Dairokuten during the period of 28 July to 11 August, are not seismogenic effects, but they may be local noises having nothing to do with the EQ (probably manmade composite noise). Finally, we go to the VHF data observed at Kunimi (in red). As compared with the situation after the EQ, the VHF noise intensity is considerably more intense. The temporal evolutions of multi-fractality parameters indicate that there are a few periods with significantly enhanced value of $w$ only before the EQ; that is, 31 July to 3 August, 8-10 August and 12 to 15 August just before the EQ. During these periods, $\alpha_{\max }$ is considerably increased $\alpha_{\max } \cong 2.5-3.0$, suggesting that the Earth's crust appears more complex, with a wider range of possible fractal exponents. This effect is most clearly recognized in the plot of the most sensitive parameter, $\Delta$ (non-uniformity factor). The VHF emissions during these periods with enhancement are likely to reflect the SOC process in the lithosphere near Kunimi. Furthermore we note that this $\Delta$ value has relaxed to the normal (or background) value after the EQ. This result appears to be consistent with the well-known result on the transition from mono- to multi-fractal behavior in the 
percolation model of breakdown at the last stage of the fracture process. This is strongly indicating that the VHF emissions observed at Kunimi (in red) may be electromagnetic precursor of the EQ, which is reflecting the nonlinear SOC behavior taking place only just around the station of Kunimi.

We here comment on some other factors, including (1) lightning and (2) magnetospheric (solar-terrestrial) effect. In the top panel in Fig. 4 we have studied the lightning effect and it is not found to be so influential. As for the second effect, (ii) magnetic activity, we looked at the temporal variation of Dst and $\Sigma K p$, and have found that the magnetic activity was not so active during the whole period, except a large geomagnetic storm on 24 August. Even this storm has not made any significant effect on VHF noise.

\subsection{Multi-fractal analysis (WTMM)}

This WTMM (Wavelet Transform Modulus Maxima) is based on continuous wavelet transform (Muzy et al., 1993). Wavelet analysis is a powerful multiscale resolution technique, well suited to understanding deeply the complex features of real world processes: "kinds" of (multi)fractality, long-range dependence, non-stationarity, oscillatory behaviour and trends. The wavelet transform is a convolution product of the data sequence (a function $f(x)$, where $x$ referred to in this study as "position", is usually a time or space variable) with the scaled and translated version of the mother wavelet (basis function), $\psi(x)$. The scaling and translation are performed by two parameters; the scale parameter $s$ stretches (or compresses) the mother wavelet to the required resolution, which the translation parameter $b$ shifts the basis function to the desired location:

$$
(W f)(s, b)=\frac{1}{s} \int_{-\infty}^{+\infty} f(x) \psi *\left(\frac{x-b}{s}\right) d x,
$$

where $s, b$ are real, $s>0$ for the continuous version (CWT) and $\psi$. The wavelet transform acts as a microscope: it reveals more and more details while going towards smaller scales, that is, towards smaller-values. One can associate with a mother wavelet a purely periodic signal of frequency $F C$ which "captures" its main oscillations $\left(F_{C}\right.$ is the center frequency of the mother wavelet). Then, it follows that the frequency corresponding to a certain scale $s$ can be expressed as $F s=(S P \times F c) / s$, where $S P$ is the sampling period. As in most wavelet-based multifractal studies and for simplicity, we use 'scale' rather than "frequency" throughout this paper.

The mother wavelet $(\psi(x))$ is generally chosen to be well localized in space (or time) and frequency. Usually, $\psi(x)$ is only required to be of zero mean, but for the particular purpose of multifractal analysis $\psi(x)$ is also required to be orthogonal to some low order polynomials, up to the degree $n-1$ (i.e., to have $n$ vanishing moments):

$\int_{-\infty}^{+\infty} x^{m} \psi(x) d x=0, \quad 0 \leq m<n$.
Thus, while filtering out the trends, the wavelet transform reveals the local characteristics of a signal, and more precisely its singularities (The Hölder exponent can be understood as a global indicator of the local differentiability of a function). By preserving both scale and location (time, space) information, the CWT is an excellent tool for mapping the changing properties of non-stationary signals. A class of commonly used real-valued analyzing wavelets, which satisfies the above condition, is given by the successive derivatives of the Gaussian function:

$\psi^{(N)}(x)=\frac{d^{N}}{d x^{N}} e^{-x^{2} / 2}$,

for which $n=N$. In this study, the analyzing wavelet is the second derivative of the Gaussian. The computation of the CWT was carried out in the frequency domain, by using the fast Fourier transform. The time-series were padded with zeros up to the next power of two to reduce the edge distortions introduced by the Fourier transform, which assumes the data are infinite and cyclic, (Torrence and Compo 1998).

It can be shown that the wavelet transform can reveal the local characteristics of $f$ at a point $x_{0}$. More precisely, we have the following power-relation:

$W^{(n)} f\left(s, x_{0}\right) \sim|\mathrm{s}|^{h\left(x_{0}\right)}$

where $h$ is the Hölder exponent (or singularity strength). The symbol " $(n)$ ", which appears in the above formula, shows that the wavelet used $(\psi(x))$ is orthogonal to polynomials up to degree $n-1$. The scaling parameter (the so-called Hurst exponent) estimated when analysing time-series by using "monofractal" techniques is a global measure of selfsimilarity in a time-series, which the singularity strength $h$ can be considered a local version (i.e. it describes 'local similarities') of the Hurst exponent. In the case of monofractal signals, which are characterized by the same singularity strength everywhere ( $h(x)=$ constant), the Hurst exponent equals $h$. Depending on the value of $h$, the input series could be long-range correlated $(h>0.5)$, uncorrelated $(h=0.5)$ or anticorrelated $(h<0.5)$.

The continuous wavelet transform described before is an extremely redundant representation, too costly for most practical applications. To characterize the singular behaviour of functions, it is sufficient to consider the values and position of the WTMM (Mallat and Hwang, 1992). The wavelet modulus maximum is a point $\left(s_{0}, x_{0}\right)$ on the scale-position plane, $(s, x)$, where $\left|W f\left(s_{0}, x\right)\right|$ is locally maximum for $x$ in the neighborhood of $x_{0}$. These maxima are located along curves in the plane $(s, x)$. The WTMM representation has been used for defining the partition function-based multifractal formalism (Muzy et al., 1994; Arneodo et al., 1995).

Let $\left\{u_{n}(s)\right\}$, where $n$ is an integer, be the position of all local maxima at a fixed scale $s$. By summing up the $q$ 's power of all these WTMM, we obtain the partition function $Z$ :

$Z(q, s)=\sum_{n}\left|W f\left(u_{n}, s\right)\right|^{q}$ 

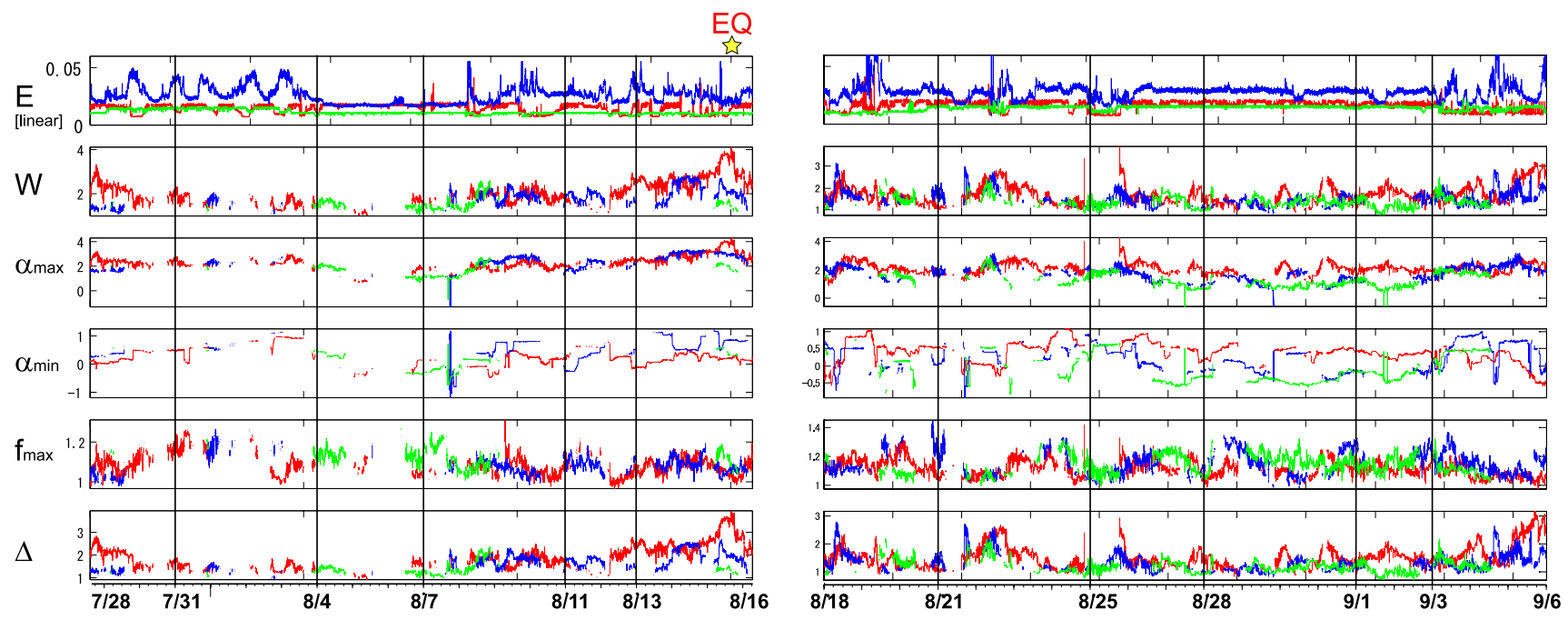

Fig. 5. The same as Fig. 5, but with the different analysis method of MF-WTMM.

By varying $q$ in this equation, it is possible to characterize selectively the fluctuations of a time-series: positive $q$ 's accentuate the 'strong' inhomogeneities of the signal, while negative $q$ 's accentuate the 'smoothest' ones. In this work, we have employed a slightly different formula to compute the partition function $\mathrm{Z}$ by using the 'supremum method' which prevents divergences from appearing in the calculation of $Z(q, a)$, for $q<0$ (e.g. Arneodo et al., 1995).

Often scaling behaviours is observed for $Z(q, s)$ and the spectrum $\tau(q)$, which describes how $Z$ scales with $s$, can be defined:

$Z(q, s) \sim \mathrm{s}^{\tau(\mathrm{q})}$

If the $\tau(q)$ exponents define a straight line, the analysed signal is a monofractal; otherwise the fractal properties of the signal are inhomogeneous, that is, they change with location, and the timeseries is a multifractal. By applying the Legendre transformation to $\tau(q)$ we can obtain the multifractal spectrum $D(h) . D(h)$, known also as the singularity spectrum, captures how "frequnecy" a value $h$ is found. After having the portition function $Z(q, s)$ including the previous DFA $Z(q, s)$, we take the same procedure to obtain the final curve $f(\alpha)$.

Figure 5 is the result based on the WTMM analysis. The format is exactly the same as in Fig. 4, and again Fig. 5 illustrates the temporal evolutions of VHF noise (top), $w, \alpha_{\max }$, $\alpha_{\min }, f_{\max }$, and non-uniformity factor $(\Delta)$ from the 2 nd to 6th. We have used 1024 points for the analysis in Fig. 5. Let us look at the temporal evolution of multi-fractal parameters. Again, it is easy to understand that some significant effects are noticed only at one station of Kunimi (in red). Only at this station we have recognized significant changes 3-5 days before the EQ; increases in $w, \alpha_{\max }$ and the non-uniformity factor $(\Delta)$. While, there is observed no significant change in $\alpha_{\text {min }}$. After the EQ, we notice no such conspicuous changes in the multi-fractal parameters.

\section{Comparison of results by different methods}

Three different methods of fractal analysis have been applied to the VHF electromagnetic noise data observed at several stations in the Tohoku area of Japan for a selected large EQ on 16 August, $2005(M=7.2)$. The first method is (1) spectrum slope analysis and (2) multi-fractal analysis with two different techniques (detrended fluctuation analysis (DFA) and wavelet transform modulus maxima (WTMM)). A comparison of the results by these methods yields the following conclusion.

1. The most important feature common to these three methods, is that significant changes are observed just during the EQ, but mainly before the EQ.

2. The above property is observed only at one station of Kunimi.

The use of only one analysis method does not enable us to make a definite conclusion on the process taking place in the lithosphere, but the simultaneous use of a few methods and the the corresponding consistence of the results by those methods, will lead us to the definite conclusion that a kind of SOC process is taking place in the VHF electromagnetic data, but not at all stations but only at a particular station (Kunimi) in this paper.

Unlike the seismogenic ULF emissions which are believed to be generated near the focal region of an EQ, VHF emissions are expected to be heavily damped during the propagation in the lithosphere, and so they are considered to be generated near the Earth's surface or in the atmosphere as a 


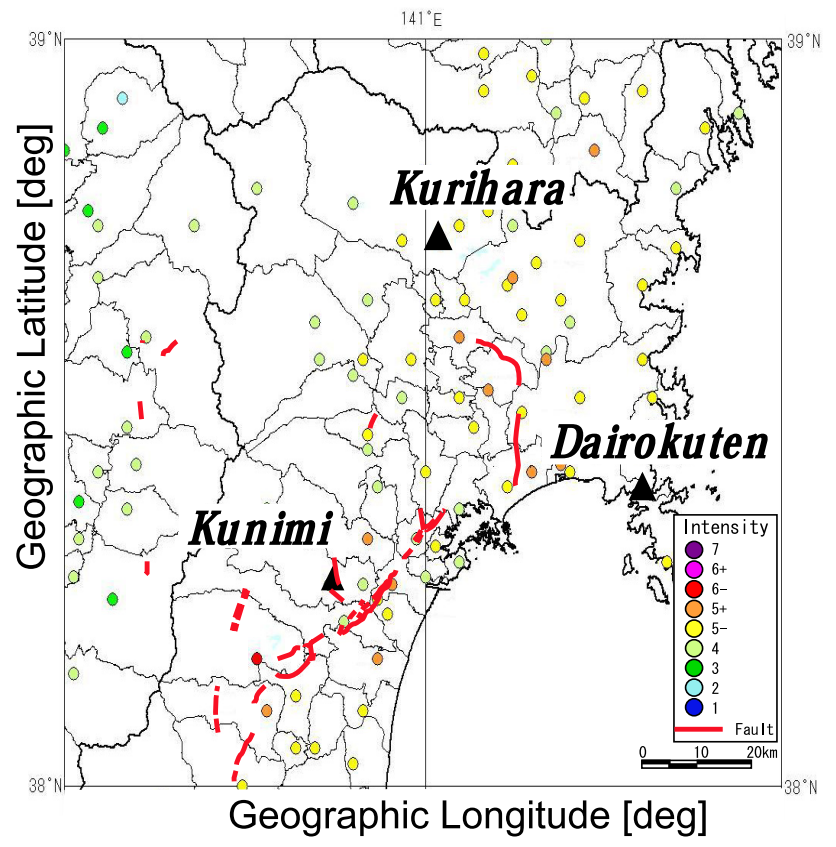

Fig. 6. Geographic map of earthquake intensity, and the fault is indicated by dotted lines.

secondary effect of precursory tectonic effects near the Kunimi station. As summarized above, we have found significant changes only at a station of Kunimi. A sharp contrast of multi-fractal parameters before and after the EQ is strongly suggesting that some SOC process is going on only at $\mathrm{Ku}$ nimi, but not at the other two stations. A few days before the EQ we have observed seemingly significant changes in multi-fractal parameters (especially $\Delta$ ), which would be a precursor to the earthquake. The change in multi-fractality before the EQ is characterized by (1) a decrease in the value of $\alpha_{\min }$, and (2) a drastic increase in the value of $\alpha_{\max }$ (correspondingly a significant increase in $w$ ). These result in a drastic enhancement in the value of $\Delta$ (non-uniformity factor). For a monofractal, $\Delta$ should be close to zero, while $\Delta$ may theoretically assume any real value $>0$ for a multifractal. Changes of the capacity dimension $D_{0}=f_{\max }$ are taken care of by the normalization by the denominator, so that $\Delta$ is really comparable throughout different time windows. Although $\Delta$ can, of course, not completely describe the $f(\alpha)$ spectra, it is very convenient for the study. This value of $\Delta$ is found to exhibit a significantly high value approaching 2.0 before the EQ (i.e., a support that the system showed a transition from homogenous to heterogeneous (from monofractal to multifractal)). This may be an important precursor to this EQ.

As is mentioned in the Introduction, the VHF noise at a particular station is composed of a few possible noise sources including (1) atmospheric noise (due to lightning), (2) manmade composite noise, (3) solar-terrestrial effect (noise from Earth's ionosphere/magnetosphere, galactic noise), and (4) any other possible noise like seismogenic emission. We have examined the first and third effects, and they are concluded not to be significant. So the possible candidates are (2) manmade composite noise without any effect of SOC and (4) lithospheric emissions in possible relation to the SOC process. We have found the significant SOC characteristics only at Kunimi, which means that the main contribution there was of the fourth candidate, lithospheric VHF emission seeming to exhibit a SOC process. On the other hand, we can conclude that there seems to be extremely small contribution of lithospheric effect, at least, at the other two stations.

The presence of precursory VHF emissions only around Kunimi and the absence of seismic effects at Dairokuten and Kurihara, might be closely related with the geological structures (including faults information) around those stations. The seismological observation after the EQ has indicated in Fig. 6 that the strongest EQ intensity was observed at the EQ shock around the station of Kunimi, and Dairokuten had the smallest intensity because of the strong rock structure below the station. We have also studied the fault region in this area, which indicates that there are fault regions named Nagamachi-Rifu fault regions, being coincident with the distribution of higher EQ intensity. Because of this geological structure beneath the station of Kumini, VHF electromagnetic emissions tend to easily appear in the atmosphere, to be observed only at this station.

Acknowledgement. This work is, in part, supported by NiCT ( R and $\mathrm{D}$ promotion scheme funding international joint research) for its supports, to which we are grateful.

Edited by: P. F. Biagi

Reviewed by: two anonymous referees

\section{References}

Arneodo, A., Bacry, E., and Muzy, J. F.: The thermodynamics of fractals revisited with wavelets, Physica A, 213, 232-275, 1995.

Degauque, P. and Hamelin, J. (Eds.): Electromagnetic Compatibility, Oxford Univ. Press, Oxford, p. 652, 1993.

Eftaxias, K., Kapiris, P., Dologlou, E., Kapanas, J., Bogris, N., Antonopoulos, G., Peratzakis, A., and Hadjicontis, V.: EM anomaly before the Kozani earthquake: A study of their behavior through laboratory experiments, Geophys. Res. Lett., 29, 1228, doi:10.1029/2001GL013786, 2002.

Fenoglio, M. A., Johnston, M. J. S., and Byerlee, J. D.: Magnetic and electric fields associated with changes in high pore pressure in fault zones application to the Loma Prieta ULF-emissions, J. Geophys. Res., 100, 12 951-12 958, 1995.

Goltz, C.: Fractal and Chaotic Properties of Earthquakes, Springer Verlag, Berlin, 1997.

Gotoh, K., Hayakawa, M., and Smirnova, N.: Fractal analysis of the geomagnetic data obtained at Izu peninsula, Japan in relation to the nearby earthquake swarm of June-August 2000, Nat. Hazards Earth Syst. Sci., 3, 229-236, 2003, http://www.nat-hazards-earth-syst-sci.net/3/229/2003/. 
Gotoh, K., Hayakawa, M., Smirnova, N. A., and Hattori, K.: Fractal analysis of seismogenic ULF emissions, Phys. Chem. Earth, 29, 419-424, 2004

Hayakawa, M. and Fujinawa, Y. (Eds.): Electromagnetic Phenomena Related to Earthquake Prediction, Terra Sci. Pub. Co., Tokyo, 667 pp., 1994.

Hayakawa, M. (Ed.): Atmospheric and Ionospheric Electromagnetic Phenomena Associated with Earthquakes, Terra Sci. Pub. Co., Tokyo, 996 pp., 1999.

Hayakawa, M., Itoh, T., and Smirnova, N.: Fractal analysis of ULF geomagnetic data associated with the Guam earthquake on August 8, 1993, Geophys. Res. Lett., 26, 2797-2800, 1999.

Hayakawa, M. and Molchanov, O. A. (Eds.): Seismo Electromagnetics: Lithosphere - Atmosphere - Ionosphere Coupling, TERRAPUB, Tokyo, 477 pp., 2002.

Ida, Y., Hayakawa, M., Adalev, A., and Gotoh, K.: Multifractal analysis for the ULF geomagnetic data during the 1993 Guam earthquake, Nonlin. Processes Geophys., 12, 157-162, 2005, http://www.nonlin-processes-geophys.net/12/157/2005/.

Ida, Y. and Hayakawa, M.: Fractal analysis for the ULF data during the 1993 Guam earthquake to study prefracture criticality, Nonlin. Processes Geophys., 13, 409-412, 2006, http://www.nonlin-processes-geophys.net/13/409/2006/.

Ida, Y., Hayakawa, M., and Gotoh, K.: Multifractal analysis for the ULF geomagnetic data during the Guam earthquake, IEEJ Trans. Fundamentals and Materials, 126, 215-219, 2006.

Kantelhardt, J. W., Zschiegner, S. A., Bunde, E. K., Bunde, A., Havlin, S., and Stanley, H. E.: Multifractal detrended fluctuation analysis of nonstationary time series, Physica A, 316, 87-114, 2002.

Kapiris, P., Polygiannakis, J., Peratzakis, A., Nomicos, K., and Eftaxias, K.: VHF-electromagnetic evidence of underlying preseismic critical stage, Earth Planets Space, 54, 1237-1246, 2002.
Kapiris, P. G., Eftaxias, K. A., and Chelidze, T. L.: Electromagnetic signature prefracture criticality in heterogeneous media, Phys. Rev. Lett., 92, 065702, 2004.

Mallat, S. and Hwang, W. L.: Singularity detection and processing with wavelets, IEEE Trans. Information Theory, 38, 617-643, 1992.

Molchanov, O. A. and Hayakawa, M.: Generation of ULF electromagnetic emissions by microfracturing, Geophys. Res. Lett., 22, 3091-3094, 1995.

Muzy, J. F., Bacry, E., and Arneodo, A.: Multifractal formalism for fractal signals: The structure-function approach versus the wavelet-transform modulus-maxima method, Phys. Rev., E 47, 875-884, 1993.

Smirnova, N., Hayakawa, M., Gotoh, K., and Volobuev, D.: Scaling characteristics of ULF geomagnetic field at the Guam seismoactive area and their dynamics in relation to the earthquake, Nat. Hazards Earth Syst. Sci., 1, 119-126, 2001, http://www.nat-hazards-earth-syst-sci.net/1/119/2001/.

Smirnova, N., Hayakawa, M., and Gotoh, K.: Precursory behavior of fractal characteristics of the ULF electromagnetic fields in seismic active zones before strong earthquakes, Phys. Chem. Earth, 29, 445-451, 2004.

Torrence, C. and Compo, G. P.: A practical guide to wavelt analysis, B. Am. Meterol. Soc., 79(1), 61-78, 1998.

Yonaiguchi, N., Ida, Y., Hayakawa, M., and Masuda, S.: Fractal analysis for VHF electromagnetic noises and the identification of preseismic signature of an earthquake, J. Atmos. Solor-terr. Phys., in press, 2007.

Varotsos, P.: The Physics of Seismic Electric Signals, TERRAPUB, Tokyo, 338 pp., 2005. 